\title{
L'approche « consciente et contrastive » de Fedor Buslaev, une innovation dans l'enseignement des langues en Russie du XIXe siècle
}

\author{
Nadéjda Kriajeva
}

\section{OpenEdition \\ Journals}

\section{Édition électronique}

URL : https://journals.openedition.org/dhfles/4461

DOI : $10.4000 /$ dhfles.4461

ISSN : 2221-4038

Éditeur

Société Internationale pour l'Histoire du Français Langue Étrangère ou Seconde

Édition imprimée

Date de publication : 1 décembre 2017

Pagination : 61-81

ISSN : 0992-7654

Référence électronique

Nadéjda Kriajeva, «L'approche « consciente et contrastive » de Fedor Buslaev, une innovation dans l'enseignement des langues en Russie du XIXe siècle », Documents pour l'histoire du français langue étrangère ou seconde [En ligne], 58-59 | 2017, mis en ligne le 10 mai 2018, consulté le 26 mars 2023. URL : http://journals.openedition.org/dhfles/4461 ; DOI : https://doi.org/10.4000/dhfles.4461

Ce document a été généré automatiquement le 26 mars 2023

Tous droits réservés 


\title{
L'approche « consciente et
} contrastive » de Fedor Buslaev, une innovation dans l'enseignement des langues en Russie du XIXe siècle

\author{
Nadéjda Kriajeva
}

\section{Les langues dans l'instruction publique : place honorable, gestion difficile}

1 Le système de l'instruction publique en Russie, forgé de toutes pièces au début du XIX siècle, de l'école primaire aux universités, se dote en même temps du Ministère qui va gérer dorénavant cet ensemble complexe dont l'importance pour la société russe augmentera de manière spectaculaire tout au long du XIX siècle $^{1}$. Les trois gymnases déjà existants (auprès des universités de Moscou, de Kazan et de l'Académie des sciences de Saint Pétersbourg), et quelques-uns nouvellement créés, obtiennent tous le statut d'établissements d'enseignement secondaire principaux, accessibles dans un premier temps à toutes les conditions sociales (excepté les serfs). De même le Ministère prenait sous sa tutelle les établissements fermés de la noblesse : corps des cadets et instituts de jeunes filles nobles, ainsi que de nombreux pensionnats privés, tenus en principe par les pédagogues étrangers. Les gymnases de filles, créés au milieu du siècle et gérés par le Cabinet de l'impératrice Marie, les séminaires du Saint-Synode, des écoles techniques et militaires complètent le paysage assez bigarré de l'enseignement secondaire au XIX ${ }^{\mathrm{e}}$ siècle.

2 Ce système a subi plusieurs réformes $(1828 ; 1849 ; 1864 ; 1872 ; 1890)$ qui modifiaient les objectifs de l'instruction, la structure, les noms des établissements et le contenu des programmes. Elles limitaient ou élargissaient à plusieurs reprises l'accès à l'éducation de différents groupes sociaux, dans le contexte politique et social instable de ce siècle : les années libérales alternaient avec les périodes d'endurcissement et de 
conservatisme. Malgré les aléas des réformes, les gymnases représentaient le noyau dur du système de l'enseignement secondaire ayant le triple objectif de préparer la jeunesse aux études universitaires, aux fonctions administratives étatiques et à l'enseignement dans les écoles inférieures.

Entre 1804 et 1828, le contenu de l'enseignement dans les gymnases est " encyclopédique $~^{2}$; il inclut de nombreuses matières étudiées en espace de quatre ans. Les gymnases font suite aux écoles de premier degré en remplaçant ainsi celles de deuxième degré mises en place dans les années $70 \mathrm{du}$ XVIII ${ }^{\mathrm{e}}$ siècle.

Depuis la réforme de 1828 et jusqu'à 1864, les gymnases ont sept classes au lieu de quatre, ils deviennent ainsi indépendants de l'enseignement des écoles de premier degré qui sont destinées désormais aux classes populaires. En même temps, se manifeste la volonté de variation des contenus : classique (deux langues anciennes), semi-classique (une langue ancienne et deux modernes) et enfin, réel (pas de langues anciennes, une langue moderne). Les distinctions établies au départ comme options (à partir de la quatrième année d'études) au sein du même établissement, donnent assez vite lieu à la création des gymnases de trois types : classique, général et réal ${ }^{3}$. L'accès à l'université et aux études supérieures, en général, est ouvert en principe aux sortants de tous les gymnases.

5 Dès 1872, la bifurcation classique/réel devient définitive, elle est très stricte. Plusieurs historiens y voient une décision politique (Alešincev 1912; Ganelin 1950; Puškarev 1956). Imposée par la volonté du ministre Dimitri Tolstoï (1823-1889), contrairement à l'opinion de la majorité des décideurs, pour contenir l'implication des jeunes à des mouvements révolutionnaires et les séparer. Désormais, les études universitaires ne sont accessibles qu'aux élèves ayant appris l'une des langues anciennes (le latin ou le grec) et non aux sortants d'établissements secondaires de type moderne: "écoles réales $»^{4}$.

6 Le résultat est au premier abord paradoxal par rapport aux tendances de la même époque dans les pays occidentaux. En effet, les humanités importées de l'Europe occidentale à l'école secondaire russe en guise de «fondement de la connaissance et de la culture générale » ont fait le chemin du " classicisme doux et réservé » des réformes $(1828 ; 1864)$ à celui qu'on appelait « dur » ou même « enragé » ${ }^{5}$ du dernier tiers du XIX ${ }^{e}$ siècle. Pourtant cela n'a pas entravé le développement du réalisme dans l'instruction publique : le réseau de type réel s'élargit et accumule de nombreux progrès.

7 Or, le trait commun de toutes les réformes de l'école secondaire dans la Russie du XIX siècle est l'importance qu'elle attribuait à l'enseignement des langues étrangères : anciennes (latin et le grec), et modernes (français et allemand). C'est aussi son trait particulier par rapport aux pays occidentaux de la même époque. La place des langues fut honorable en tout type de gymnases, elles y occupaient entre quarante et soixante pour cent de la totalité des cours dispensés. Les langues modernes étant prioritaires dans l'enseignement encyclopédique du début du XIX ${ }^{e}$ siècle ont progressivement fait la place à la langue maternelle d'abord, et ensuite ont cédé la priorité aux langues anciennes (mais seulement dans le contenu désigné comme classique). En effet, la langue russe en tant que matière d'enseignement ne commence à renforcer radicalement ses positions dans les gymnases que vers le milieu du siècle sur le fond du nationalisme grandissant ${ }^{6}$. Elle se voit attribuer de quatre à deux leçons hebdomadaires comme chacune des langues vivantes d'ailleurs ${ }^{7}$. Le grec ancien introduit et le latin 
renforcé par la réforme de 1828 obtiendront en 1872 jusqu'à deux tiers du temps prévu pour les langues dans les gymnases classiques avec les deux langues anciennes.

8 Néanmoins, le "poids" de chacune des langues et leur rôle en tant que moyen d'instruction de jeunes esprits, la répartition des leçons, les méthodes d'enseignement préoccupent fortement les milieux engagés de façon différente dans le processus d'organisation et le fonctionnement des gymnases: décideurs et concepteurs de programmes, universitaires et enseignants mêmes de ces établissements. En effet, la question des langues dans les gymnases fait partie des discussions fondamentales portant sur la nature de l'enseignement à privilégier par l'école secondaire: «classique » (humaniste) ou "réaliste». Or, parmi les conditions préalables qui ont rendu possibles ces débats, nous mentionnons en premier lieu les activités du Comité Scientifique fonctionnant dès la création du Ministère de l'Instruction publique. Il a été chargé de la rédaction des programmes et de l'examen de différents outils didactiques conçus à l'usage dans les établissements éducatifs ${ }^{8}$. L'édition périodique du Ministère de l'Instruction publique existant depuis 1804 et transformée en 1834 en un mensuel officiel, devient la scène la plus importante de débats pédagogiques en Russie. De même, plusieurs périodiques créés à des moments différents du XIX ${ }^{\mathrm{e}}$ siècle suivaient les activités bouillonnantes du domaine de l'instruction publique et la polémique des partis opposés, confrontant ainsi les idées de différents acteurs de l'instruction publique sur l'éducation de la jeunesse ${ }^{9}$. Ils publient les différentes réactions aux projets de 1857, 1861, 1863 et de 1871 soumis pour discussion au niveau national ; ainsi que les opinions des savants et pédagogues allemands et français sollicités par le comité scientifique $^{10}$. Remarquables sont les publications de la section des langues auprès du Musée pédagogique militaire (1885-1915) sur la méthodologie directe et son application aux conditions de l'école secondaire en Russie ${ }^{11}$.

9 À l'aide de ces sources, dont plusieurs sont numérisées assez récemment ${ }^{12}$, on arrive dans un premier temps à une constatation bien banale d'ailleurs : jusqu'au milieu du $\mathrm{XIX}^{\mathrm{e}}$ siècle dans les gymnases russes règne la méthodologie grammaire/traduction ${ }^{13}$, les cours systématiques de grammaire et les dictionnaires étant utilisés dans toutes les classes en guise d'outils didactiques principaux ${ }^{14}$. Ainsi, les élèves se trouvaient confrontés depuis le début de leur scolarité à l'apprentissage simultané de plusieurs langues: le russe avec le slavon d'église; deux langues anciennes et deux modernes (français et allemand), ayant en tout six grammaires différentes en main.

10 Mentionnons quand même que les enfants qui entraient dans des gymnases appartenaient aux familles nobles ayant des précepteurs; donc, à l'âge de dix ans ils savaient lire et écrire en russe et en français (Ihnatovicz 1887 : IX). Cela rendait possible l'usage des manuels de grammaire en principe dès la première année d'études, mais ne facilitait guère l'apprentissage linguistique représentant une espèce de «torture grammaticale » permanente et peu efficace.

11 Cet état des choses déplorable dans l'enseignement des langues à l'école publique donne lieu à une critique virulente de plus en plus intense surtout à partir des années trente. Les slavophiles partisans d'un courant de pensée russe radical accusaient les savants russes de "décrire la langue maternelle à travers les lunettes étrangères ", suivant le modèle des grammaires latines ${ }^{15}$. Même les auteurs reconnus pour l'originalité de leurs travaux sur la langue russe, comme Nikolaï Greč (1787-1867) et Alexandre Vostokov (1781-1864), n'ont pas été épargnés de ces reproches, parce que leurs adaptations de grammaire russe à l'usage scolaire gardaient en principe la 
structure de description traditionnelle: lettres/sons, syllabes, parties du discours; déclinaison, conjugaison ${ }^{16}$, etc.). Les discussions plus généralistes, sur le contenu « réaliste " et/ou "classique " (humaniste) de l'enseignement à dispenser dans les gymnases, amplifient la problématique de l'enseignement des langues durant la seconde moitié du XIXe siècle (Grot 1871; Feoktistov 1872: 1-33; Hippeau 1878; Alešincev 1912).

Or, l'enseignement/apprentissage du russe a été pris pour cible en premier lieu, celui des autres langues n'étant pas été touché de la même façon: on lui reprochait la mauvaise répartition du temps entre les langues et le rôle exagéré /insuffisant dans l'éducation.

\section{Réflexions d'un « éclaireur " sur l'essence de l'enseignement linguistique à l'école et la complémentarité des langues}

13 C'est dans ce contexte difficile qu'apparaît l'ouvrage de Fedor Buslaev intitulé modestement "À propos de l'enseignement de la langue maternelle » $(1844)^{17}$. Le jeune auteur (il a 26 ans à l'époque) le présente comme un bilan de réflexions issu de ses lectures sur le sujet qu'il confronte aux problèmes d'enseignement de la langue russe rencontrés par lui-même.

Buslaev Fedor Ivanovič (1818-1897). Linguiste, historien, pédagogue, professeur de l'Université de Moscou, membre de l'Académie Impériale des sciences de Russie (1860), vice-président en 1889. Après avoir terminé le gymnase dans une ville provinciale (Penza), il entre à l'université de Moscou pour une formation en philosophie et philologie (1834-1838). Entre 1838-1841, il séjourne dans plusieurs pays (Allemagne, Suisse, France, Italie) en tant que précepteur des enfants du comte Stroganov S.G. (1794-1882), directeur du district éducatif de Moscou. Pendant cette période Buslaev découvre et s'inspire des idées linguistiques de Wilhelm von Humboldt et de Jacob Grimm, de même il étudie la pédagogie suisse et allemande (Johann Pestalozzi, Adolphe Diesterweg, Karl Mager, Johann Deinhardt, Friedrich Günther, etc.) Peu après son retour à Moscou, enrichi de nouvelles connaissances, Buslaev débute sa carrière universitaire (1842), en se consacrant à l'étude historique des langues. En parallèle, le jeune savant enseigne la langue russe dans des établissements secondaires de Moscou (1841-1849) et, enfin, il est choisi comme enseignant de langue et littérature russes pour les enfants de la famille impériale (1859-1861). Comparatiste remarquable et historien de la langue russe, il a toujours cherché à rapprocher l'école et la science, ayant jeté les bases de la didactique moderne de la langue maternelle. Ses principaux ouvrages dans ce domaine, À propos de l'enseignement de la langue maternelle (1844), Essai de grammaire historique de la langue russe (1858), Manuel de la grammaire russe comparée à la grammaire du slavon d'église (1870), "...ont ouvert une nouvelle époque dans l'enseignement de la langue maternelle » jetant les bases scientifiques de sa didactique (Grot $1876: 195)$.

15 La première partie du livre incluant plusieurs chapitres commence par une constatation de nombreuses insuffisances de l'enseignement des langues : maternelle et étrangères à l'école et l'analyse des théories en matière de l'éducation de plusieurs savants et pédagogues allemands et suisses. L'auteur passe en revue les arguments des 
deux "parties pédagogiques" opposées : humanistes et réalistes, en insistant sur le caractère intransigeant et inflexible de leur argumentation. Pourtant, selon lui, un compromis entre ces partis est possible s'ils arrivent à trouver un accord sur le vrai rôle que la langue maternelle doit jouer dans l'éducation (Buslaev 1844 : 34-42).

Dans le chapitre "Méthode de l'enseignement de la langue russe", Buslaev expose des fondements de sa nouvelle approche qu'il baptise de consciente et pratique, contrastive et comparée. Les bases de ses innovations sont linguistiques, psychologiques et pédagogiques. Avant tout, dit-il; il ne faut pas confondre les objectifs scientifiques et scolaires de l'étude de la langue maternelle, ils sont différents et ne peuvent pas être atteints par les mêmes moyens. À l'appui de la pensée de Grégoire Girard (1765-1850), pédagogue suisse affirmant que «tout manuel et toute matière enseignée doivent être adaptés aux objectifs éducatifs, à l'âge et aux capacités intellectuelles des élèves " (Girard, 1827), Buslaev essaie de démontrer que les manuels de langue russe de son temps, «bourrés de terminologies" incompréhensibles aux jeunes enfants, ne correspondent point à ces exigences :

Dès le plus jeune âge chaque Russe sait déjà décliner et conjuguer, il ne se trompera pas en accords et en régime des mots, à l'exception de quelques cas particuliers, d'ailleurs c'est seulement ces derniers qui devraient figurer dans le manuel. Il est inutile que les enfants sachent les termes d'étymologie, de syntaxe, d'orthographe dont les manuels sont remplis avant qu'ils n'aient compris ce qui est la proposition (phrase) et ses parties, ou la signifiance des différentes espèces de mots, avant qu'ils n'apprennent à saisir avec facilité le sens de ce qu'ils lisent et à le relater par écrit. (Buslaev $1844: 30)^{18}$

17 Buslaev précise le sens spécifique qu'il donne aux termes de langue et de grammaire. Influencé par la philosophie du langage de Wilhelm von Humbold (1767-1835) il fait la distinction entre langue produit et langue activité, et respectivement entre les approches philologique et linguistique. De ce point de vue, avec le terme de grammaire on peut désigner l'arsenal de tous les éléments constituant une langue mais aussi son mécanisme en action, en construction permanente des sens.

Par conséquent, dit-il, deux manières d'enseigner la grammaire sont possibles. La première, très répandue, dresse l'inventaire des divers éléments dont la langue est constituée : sons/lettres, syllabes, parties du discours avec leurs accidents, c'est une approche philologique. Tout ouvrage de grammaire systématique en incarne l'exemple. Cette façon d'enseigner ne peut être que difficile et inefficace à l'école.

La seconde, approche linguistique, encore très rare à l'école, s'appuie sur l'activité langagière de l'enfant en cherchant à développer son « don inné » de la parole. C'est en cours de conversation et de lecture, à travers la phrase, unité principale de la parole, que les élèves guidés intelligemment par le maître, découvrent la grammaire de leur langue maternelle et la richesse de son vocabulaire.

20 Pour pouvoir appliquer l'approche linguistique à l'enseignement du russe il est nécessaire dit-il, de tenir compte de plusieurs évidences pédagogiques :

Vers l'âge de dix ans la grammaire de la langue maternelle (en tant que mécanisme) est acquise : tout enfant russe sait s'exprimer en russe sans se tromper dans le choix des formes grammaticales, mais cette maitrise est inconsciente. Pour qu'elle devienne consciente, il faut aider l'enfant à observer et à analyser son propre usage et celui des autres.

Développer les capacités d'expression orale et enrichir le vocabulaire des élèves est l'objectif principal de l'enseignant à ce stade. Cela permettra la progression dans la lecture qui nécessite comme pré- requis une bonne pratique du langage à l'oral. 
Il est logique de partir d'une maîtrise inconsciente du langage vers sa maîtrise consciente par plusieurs étapes: développer l'activité langagière des élèves en conversant et en lisant avec eux, leur expliquant peu à peu le fonctionnement de phénomènes linguistiques, et les faire progresser sur ces bases dans l'expression orale et écrite. C'est seulement après cette étape fondamentale d'apprentissage que l'étude de la langue maternelle sera accompagnée et complétée par l'usage de manuels de grammaire, adaptés aux objectifs de chaque année.

Dans ces conditions, tout ouvrage de grammaire théorique (systématique) doit être proscrit pour les trois premières années (sur sept) des gymnases; il est inutile, voire nuisible au processus de l'apprentissage (Buslaev $1844: 64-70$ ).

21 Dans la deuxième partie du livre, Buslaev décrit le déroulement d'une série de leçons de russe (niveau élémentaire) selon sa méthode. Il utilise les extraits d'un conte d'Alexandre Pouchkine, des petites histoires de la vie quotidienne, des fables, etc. De même, on y trouve des divers matériaux historiques, stylistiques, dialectaux destinés à l'usage des enseignants (Buslaev 1844 : 192-371).

Le chapitre intitulé "Les liens du russe avec les autres matières d'enseignement " contient plusieurs pages consacrées à l'enseignement des langues étrangères : anciennes et vivantes (Buslaev 1844 : 43-48; 57-64).

D’une part, Buslaev y cherche à démontrer que la pédagogie de la langue maternelle diffère fondamentalement de celle des langues étrangères :

La méthode de l'enseignement de la langue maternelle se distingue de celle qui s'applique aux langues étrangères, car elle est définie par nature même de l'objet et le développement psychologique progressif de l'enfant. La grammaire de la langue maternelle représente la logique la plus simple, avec laquelle l'enfant peut être mené, sans l'apercevoir, des choses bien simples à celles qu'on ne découvre qu'à l'aide de méthodes scientifiques... [...] Les langues étrangères s'apprennent sans cet avantage, car elles ont toujours la langue maternelle comme intermédiaire. C'est pourquoi l'analyse de la phrase russe doit précéder celle de la langue latine. Pour les avantages de cette dernière même, un certain fond du savoir grammatical doit être créé préalablement, à l'analyse des phrases de la langue maternelle. La grammaire russe servira ainsi d'intermédiaire pour la maîtrise de la grammaire latine. L'apprentissage de la grammaire d'une langue étrangère en effet est un passage au stade supérieur, théorique dans l'étude des langues. (Buslaev 1844 : 57)

De l'autre, il souligne que la différence des approches n'empêche pas la création de liens coopératifs, la complémentarité entre les langues enseignées :

C'est pour leur propre intérêt que les professeurs des langues modernes doivent soutenir l'enseignement solide des langues anciennes ainsi que l'existence des gymnases classiques, seuls garants de l'instruction dans l'esprit véritablement européen. En effet, d'une part, l'étude élémentaire comparée rapproche des langues vivantes (russe, française, allemande) à leurs « ancêtres »: le latin, le grec ancien et le slavon. D'autre part, elle met en relief les différences entre les langues vivantes mêmes par le biais de comparaison avec la langue maternelle (russe en l'occurrence). Tout cela peut et doit créer des fondements solides du savoir permettant la distinction de ce qui est commun à toute l'humanité, et du national, propre à une nation. (Buslaev $1867: 60$ )

Or, selon Buslaev, la démarche similaire serait possible dans l'apprentissage des langues vivantes à condition que les élèves aient le minimum d'expérience de communication orale dans ces langues ${ }^{19}$. Il donnera le détail de cette idée plus tard, en 1866, dans son plan d'enseignement des langues dans les établissements secondaires des filles. Il y dira que la moitié de la première année de l'apprentissage du français ou de l'allemand doit se faire sans appui à aucun texte, ni manuel de grammaire : les éléments grammaticaux 
et le vocabulaire de base seront appris en communication orale. La seconde moitié de la première année verra l'introduction de l'écriture et de la lecture de petits textes fondés sur le vocabulaire et la grammaire acquis (Buslaev $1866: 435$ ).

Buslaev souligne qu'au stade où les élèves commencent à maîtriser consciemment la langue maternelle, ils sont capables à manier avec facilité le métalangage grammatical afin d'analyser les phénomènes de nature similaire dans d'autres langues. Pour que ce métalangage soit utile, il doit constituer un ensemble cohérent et simple. Par conséquent une concertation des enseignants de toutes les langues est nécessaire. Ils doivent s'appuyer sur le métalangage grammatical acquis en cours d'apprentissage de la langue russe ${ }^{20}$. Or, Buslaev est manifestement pour l'étude des langues étrangères assez tardive: elle doit attendre que les bases conscientes de la langue maternelle soient raffermies (Buslaev 1844 : 60-65, 118-119).

Cet ouvrage, sûrement trop iconoclaste pour l'époque, n'a pas eu d'impact immédiat sur les esprits et les opinions dans les milieux éducatifs russes, étant l'objet de vives critiques à sa parution. Pourtant selon les historiens de la didactique des langues, et certains contemporains de l'auteur, ce livre avait jeté les bases de la réflexion sur le rôle fondamental de la langue maternelle dans l'éducation des enfants. Ses idées ont conquis peu à peu de nombreux partisans, parmi lesquels les acteurs influents des mouvances pédagogiques de la seconde moitié du XIX ${ }^{\mathrm{e}}$ siècle : Konstantin Ušinski (1823-1871), Vladimir Stounin (1826-1888), Vasilij Vodovozov (1825-1886), mais aussi Léon Tolstoï (1828-1910). Les collègues de Buslaev, Jakob Grot (1812-1893) et Izmail Sreznevskij (1812-1880), académiciens et universitaires, ont adhéré à ses idées en les promulguant par la suite depuis leurs universités respectives de Saint-Pétersbourg et de Kharkov (Grot 1876 ; Šaxmatov 1898 : 7-12 ; Protčenko 1992).

En fin de compte l'approche buslavienne de l'enseignement de la langue maternelle et des langues étrangères est largement reconnue dans les années soixante: elle sera officialisée dans les documents de réformes des gymnases de 1863 et 1872. Buslaev, se chargera lui-même à la demande des institutions de la rédaction de plusieurs plans et programmes d'enseignement des langues et des littératures dans les établissements secondaires (Buslaev $1866 ; 1870 ; 1886$ ). première, elle devient un vade-mecum pour les professeurs du russe, langue maternelle des élèves.

\section{L'impact de l'approche buslavienne sur l'apprentissage des langues étrangères dans les établissements d'enseignement secondaire}

La réforme de 1872 divisant définitivement les établissements secondaires en classiques (gymnases de garçons) et modernes (écoles réales ayant trois options, écoles militaires, gymnases de filles) sera à l'origine d'une bifurcation méthodologique dans l'application de la méthode consciente et contrastive aux langues étrangères. Elle est définie par les objectifs différents de l'apprentissage des langues étrangères dans ces établissements : (Orbinskij 1868 ; Radonežskij 1872 : 249-269 ; Vejsmann 1890 : 1-7 ; Varon 1874 : 58-95; Hippeau 1878). Dans les gymnases classiques les langues vivantes (français et l'allemand) sont reléguées dans le groupe de matières secondaires, elles sont 
considérées comme complémentaires pour la formation linguistique, «n'ayant pas le même potentiel que la langue maternelle et les langues anciennes» ce qui chagrine d'ailleurs des enseignants aguerris (Smirnovskij 1871; Margot 1896: V-VI). Dans les établissements non classiques, où les langues vivantes ont une place importante et ne sont pas en concurrence avec les anciennes, assumant seules la charge " contrastive " de la méthode, elles peuvent se permettre le luxe de créer le «fondement pratique initial » pour passer à l'étude consciente de la grammaire française et/ou allemande dans les classes supérieures (Varon $1874 ; 1882$; Veismann 1890).

31 Les concepteurs de manuels de langues vivantes cherchent à suivre les exigences des plans ministériels d'études. D'un côté, l'effort d'adaptation des «cours traditionnels à objectifs pratiques » créés auparavant pour l'enseignement dans les gymnases. Les titres de cours "concentriques", "gradués", "progressifs», "avec les textes suivis " vont être complétés par « comparé» au russe, au latin, au grec (Fleury 1872; 1876; 1892 ; Fenou 1871 ; Challandes 1876 ; Varon 1872 ; 1882 ; Ihnatovicz 1899 ; Raievsky 1890). De l'autre, une progression rapide de méthodes rédigées (ou adaptées à l'usage des russophones) dans l'esprit de la méthodologie directe: «Berlitz/ Robertson»; "américaine », « intuitive » (Constantin 1905; Silina 1895; Feuillye \& Martin 1904; Chabaline \& Maslof 1907).

Pourtant, ces méthodes auront un emploi spécifique et bien délimité : les premières sont en usage dans toutes les classes des gymnases classiques et les classes supérieures d'autres établissements. Les secondes sont utilisées de préférence dans les classes inférieures et moyennes des établissements non classiques. Cette règle sera de rigueur permanente: la méthodologie directe n'aura qu'une place restreinte au sein de l'approche "consciente et pratique, contrastive et comparée » dans l'enseignement secondaire en Russie vers la fin du XIX ${ }^{e}$ siècle et le début du XX ${ }^{e}$ siècle.

André Lirondelle (1879-1952), universitaire français et fonctionnaire du Ministère de l'Éducation, qui s'est intéressé en 1909 à l'enseignement du français dans les gymnases russes, le caractérisait avec justesse ainsi :

[...] Entre le système dit « Berlitz » et le système "philologique » un compromis est possible. Ce système "mixte» est employé dans les meilleurs établissements d'enseignement secondaire, à Moscou, par exemple, où j'ai été heureux de le voir fonctionner dans les gymnases VIII et IX. [...] La tendance actuelle est de ne pas appliquer intégralement la méthode naturelle tout en sauvegardant l'esprit ; les moyens fondamentaux resteront: l'enseignement par la vue, la conversation, la lecture. L'enseignement par la vue dominera dans les classes inferieures, puis des tableaux muraux, on passera aux livres. La conversation en langue étrangère est le lien qui unira toutes les classes, et sous ce nom de conversation on comprendra la conversation orale, la paraphrase, et les reproductions de récits par écrit. La traduction ne restera que comme moyen de contrôle. À partir de la $\mathrm{VI}^{\mathrm{e}}$ classe l'enseignement de la littérature s'ajoutera à celui de la langue (Lirondelle 1909: 8-10). 


\section{BIBLIOGRAPHIE}

ALEŠINCEV, Ivan (1912). Istoria gimnazičeskogo obrazovania $v$ Rossii XVIII $i$ XIX veka [Histoire de l'enseignement dans les gymnases de Russie au XVIII et XIX ${ }^{\mathrm{e}}$ siècles]. Saint-Pétersbourg : Izdanije O.Bogdanovoj.

BULIČ, Nikolaï (1902). Očerki po istorii russkoj literatury i prosvě̌čenia s načala XIX veka [Essais d'histoire littéraire et de l'instruction publique russes dès début du XIX ${ }^{\mathrm{e}}$ siècle]. T.1. SaintPétersbourg : Typographie Stasulevitch.

BUSLAEV, Fedor (1992) [1844]. O prepodavanii otečestvennogo jazyka [À propos de l'enseignement de la langue maternelle] In F. Procenkobuslaev. 0 prepodavanii otečestvennogo jazyka. Moscou : Prosveščenie, 25-371.

BUSLAEV, Fedor (1992) [1848]. Programma russkogo jazyka i slovesnosti. Sostavlena na osnovanii nastavlenija dla obrazovania vospitannikov voenno-učebnyx zavedenij [Le programme d'études de la langue et littérature russes. Pour l'éducation des élèves d'établissements secondaires militaires]. In Fedor Buslaev. O prepodavanii otečestvennogo jazyka. Moscou : Prosveščenie, 410-430.

BUSLAEV, Fedor (1858). Opyt istoričeskoj grammatiki russkogo jazyka. Učebnoe posobie dl'a prepodavatelej. Č.1. Etymologija. Č. 2. Sintaksis. [Grammaire historique de la langue russe. À l'usage des enseignants. P.1. Étymologie. P.2. Syntaxe]. Moskou : Universitetskaja tipografija. BUSLAEV, Fedor. (1992) [1866]. Obščij plan i programmy obučenia jazykam $v$ ženskix sredneučebnyx zavedeniax [Le Plan général et les programmes d'études des langues dans les établissements secondaires féminins]. In Fedor Buslaev. O prepodavanii otečestvennogo jazyka. Moscou : Prosveščenie, 431-472.

BUSLAEV, Fedor (1992) [1867]. « Otzyv professora Buslaeva F.I. o Programme russkogo jazyka I slovesnosti sostavlennoj učitelami gimnazij Moskovskogo učebnogo okruga na s'ezde $1866 \mathrm{v}$ Moskve » [L'avis du professeur Buslaev sur le Programme de langue et littérature russes, rédigé par les enseignants des gymnases du District de Moscou pour le Congrès pédagogique de 1866 à Moscou]. In Fedor Buslaev. 0 prepodavanii otečestvennogo jazyka. Moscou : Prosveščenie, 472-479.

BUSLAEV, Fedor (1870). Učebnik russkoj grammatiki, sbližennoj s cerkovno-slav'anskoju, s priloženiem obrazcov grammatičeskogo razbora. Dla srednix učebnyx zavedenij [La grammaire russe comparée à celle du slavon d'église avec les exemples d'analyse grammaticale en annexe. À l'usage de l'école secondaire]. $2^{\mathrm{e}}$ édition. Moscou : Obščestvo rasprostranenija poleznyx knig.

BUSLAEV, Fedor \& GALAXOV, Aleksej (1992) [1886]. Obščij plan i programmy obučenija jazykam $i$ literature $v$ ženskix credne-učebnyx zavedenijax [Le plan général et les programmes d'études des langues et des littératures dans les établissements secondaires de filles]. In Fedor Buslaev. 0 prepodavanii otečestvennogo jazyka. Moscou : Prosveščenie, 431-471.

CHABALINE, C. \& MASLOF, O. 1907. Phrases usuelles et dialogues destinés à faciliter l'étude de la conversation. Moscou : Škola.

CHALLANDES, Adamir (1876). Grammaire française élémentaire à l'usage des classes inférieures et moyennes des établissements d'éducation par A. Challandes maitre de langue française à la communauté impériale de demoiselles nobles. St Pétersbourg: Trenké et Fusnot.

CONSTANTIN, Aimé (1905). Cours pratique de langue française à l'usage des écoles. Ouvrage approuvé par le Ministère de l'Instruction publique, par le Comité des établissements des écoles militaires et 
par celui des Instituts et gymnases de demoiselles. $15^{\mathrm{e}}$ édition. I ${ }^{\text {ère }}$ partie. Saint-Pétersbourg : Glazounof (Collection « Méthode-Robertson »).

FEUILLYE, L. \& MARTIN, L. (1904). Nouveau cours de français : d'après les principes de la méthode directe, naturelle ou intuitive, par L. Feuillye \& L. Martin. Saint-Pétersbourg : Trenké et Fusnot.

FENOU, Nicolas (1871). Grammaire française comparée à la grammaire russe. $2^{2}$ éd. Saint-Pétersbourg : Isakov.

FEOKTISTOV, Evgenij (1872). « Otzyvy inostrannoj pečati o našej učebnoj reforme » [Notre réforme éducative à travers les opinions de la presse étrangère]. Žurnal Ministerstva Narodnogo Prosveščenija, Partie 164, 1-33.

FLEURY, Jean (1872). La Grammaire en action, cours raisonné et pratique de langue française, refondue et abrégée (édition russe-française). Saint-Pétersbourg : Tipografija Akeademiji.

FLEURY, Jean (1876). La Grammaire en action, considérablement simplifiée et accompagnée d'une traduction en russe. Saint-Pétersbourg: Trenké et Fusnot.

FLEURY, Jean (1892). La grammaire en action à l'usage des Russes par Jean Fleury. $9^{\mathrm{e}}$ édition. StPétersbourg : Trenké et Fusnot.

GANELIN, Š. I. (1950). Očerki po istorii srednej školy v Rossii vtoroj poloviny XIX veka. Moskva : Učpedgiz. GEORGIEVSKIJ, Aleksandr (1902). K istorii Učenogo komiteta [Complément à l'histoire du Comité Savant]. Saint Pétersbourg : Typographie du Sénat.

GIRARD, G. (1827). Explication du plan de Fribourg en Suisse. Lucerne : chez Xavier Meyer.

GROT, Jakob (1903) [1871]. « O klassičeskom obrazovanii. V čem suščnost' voprosa » [De l'enseignement classique. L'essence du problème]. In Karl J. Grot. (rééd.). Trudy Ja. K. Grota. SaintPétersbourg : I.N. Kušnerev et Cie. T. 5, 186-188.

GROT, Jakob (1876). «E. Belavskij. Etimologija cerkovno-slav'anskogo I russkogo jazyka, sbližennaja s etimologiej jazykov grečeskogo i latinskogo » [E. Belavskij. Étymologie du slavon d'église rapprochée de celles du grec et du latin]. Žurnal Ministerstva Narodnogo Prosveščenija, Partie 183, 194-204.

HIPPEAU, Célestin (1878). L'Instruction publique en Russie. Paris : Didier et Cie.

IHNATOVICZ, Vitold (1899). Manuel concentrique de langue française comparée au russe et au latin divisé en trois parties. Cours élémentaire ( $3^{e}$ chapitre en texte suivi). $17^{\mathrm{e}}$ édition. Saint-Pétersbourg: D. D. Polubojarinov.

KouzminA, N. (2005). « Les langues vivantes dans les établissements éducatifs russes au Siècle des Lumières. En amont de l'histoire de l'enseignement du français aux russophones ». Documents pour l'histoire du français langue étrangère ou seconde, 35, 7-26.

LIRONDELLE, André (1909). «L'enseignement des langues vivantes, et particulièrement du français, dans les établissements de l'enseignement secondaire en Russie ». Revue internationale de l'enseignement, 314-327 (tiré à part). Paris : Librairie Soufflot.

MARGOT, David (1905). Cours élémentaire et progressif de langue française à l'usage des classes inférieures et moyennes des écoles. $36^{\mathrm{e}}$ édition. Saint-Pétersbourg : Trenké et Fusnot. MARGOT, David (1896). Grammaire théorique et pratique de la langue française à l'usage des classes supérieures des écoles, par D. Margot, Directeur de l'école des paroisses réformées, et Lecteur en langue française à l'université de Saint-Pétersbourg. Ouvrage adopté par le Ministère de l'Instruction publique. $\mathrm{I}^{\text {ère }}$ partie. $7^{\mathrm{e}}$ édition. Saint- Pétersbourg: Trenké et Fusnot. 
ORBINSKIJ, Robert (1868). « O prepodavanii inostrannyx jazykov » [De l'enseignement des langues étrangères]. Žurnal Ministerstva Narodnogo Prosveščenija, Partie 137, 546-601.

PROTČENKO, Ivan (1992). «F.I. Buslaev-vydauščijs'a filolog i pedagog » [F.I. Buslaev, philologue de talent et pédagogue éminent]. In Ivan Protcenko \& Ludmila Xodakova (rééd.). F. Buslaev. 0 prepodavanii otečestvennogo jazyka. Moscou : Prosveščenie, 7-24.

PUŠKAREV, Sergej (1956). Rossia 1801-1917. Vlast' i obščestvo [La Russie en 1801-1917. Pouvoir et société]. PAris : Posev.

RAIEVSKY, Ekaterina (1890). Essai de grammaire comparée de la langue russe et française, d'après la nouvelle méthode du membre de l'Académie J. Grot. Par Madame Raievsky. Moscou : Gerbek, III.

RADONEŽSKIJ, Aleksandr (1872). « O sovmestnom prepodavanii russkogo i latinskogo jazykov v pervom klasse našix gimnazij » [De la complémentarité de l'enseignement du russe et du latin en première année d'école]. Žurnal Ministerstva Narodnogo Prosveščenija, Partie 160, 249-269.

SILINA, Aleksandra (1895). Premières lectures et leçons de causerie par Al.S. 4 édition revue et augmentée. Saint-Pétersbourg : Académie Impériale des sciences.

SILINA, Aleksandra (1902). Premières lectures et leçons de causerie par Al.S. $9^{e}$ édition revue et augmentée. À l'usage des classes inférieures des gymnases et instituts des filles. Saint-Pétersbourg: Académie Impériale des sciences (Compte rendu : Žurnal Ministerstva Narodnogo Prosveščenija, Partie 298, 1895, 10-11).

SMIRNOVSKIJ, Petr (1871). « K voprosu ob izučenii novyx jazykov v našix gimnazijax » [Au sujet de l'étude des langues étrangères vivantes dans nos gymnases). Žurnal Ministerstva Narodnogo Prosveščenija, Partie 157 /3, 45-58.

VARON, Evgenij (1874). «O prepodavanii inostrannyx jazykov voobšče I franzuzskogo jazyka v osobennosti » [À propos de l'enseignement des langues étrangères en général, et du français, en particulier]. Žurnal Ministerstva Narodnogo Prosveščenija, Partie 175, 58-95.

VARON, Eugène (1874) [1872]. Cours élémentaire méthodique et pratique de langue française à l'usage des Russes. Saint-Pétersbourg et Helsingfors : Typographie de l'Académie.

VARON, Eugène (1882). Syntaxe française à l'usage de la jeunesse russe. St-Pétersbourg : Typographie de l'Académie.

VEJSMANN, Aleksandr (1890). « K voprosu o prepodavanii novejšix jazykov francuzskogo i nemeckogo, v gimnaziax ». [Au sujet de l'enseignement des langues vivantes, française et allemande dans les gymnases]. Žurnal Ministerstva Narodnogo Prosveščenija, Partie 269 /3, 1-7.

\section{NOTES}

1. Le manifeste de l'empereur Alexandre $\mathrm{I}^{\mathrm{er}}$ de 1802 sur la création du Ministère la division du territoire de l'empire en six districts (okrugi) éducatifs sous tutelle des universités nouvellement créées. Celle de Moscou existait depuis 1755, en 1802-1804 sont fondées les universités de Kharkov, de Kazan, de Dorpat (Tartu) et de Vilna (Vilnius). Le district de Saint-Pétersbourg a été géré par l'Institut pédagogique, transformé en université dès 1819.

2. Certains historiens suggèrent plusieurs modèles occidentaux qui ont contribué à l' « encyclopédisme », au « luxe de polymathie » des premiers programmes de gymnases russes (1804), évoquant par exemple le projet de l'éducation nationale de Nicolas de Condorcet (1743-1794) débattu en 1792 à la Convention (Alešincev 1912 : 21-22; Bulič 1902 : 42-43). 
3. Ainsi, l'appellation de gymnase en russe : gimnazija dès le début de son usage avait le sens plus étendu qu'ailleurs, en désignant un établissement éducatif d'enseignement secondaire, intermédiaire entre l'école inférieure et supérieure (université, écoles supérieure spécialisées) ce qui explique la tentative d'en marquer la pré-spécialisation vis-à-vis des études supérieures: gimnazija klassičeskaja (classique), g. obščaja (commun), g. realnaja ( réal/réel ). Le dernier nom gimnazia realnaja avait été traduit en français à l'époque de deux façons : "gymnase-s réel-s » (Hippeau 1878 : 217), « gymnase-s réal/ réaux » ( Lirondelle 1909 : 3-5). C'est après la réforme de 1871-1872, qu'au sein du secondaire s'installe une opposition: entre les gymnases (donnant accès à l'université) et les écoles réales (realnye učilišča, en russe) qui n'étaient autre chose que des anciens gymnases réaux et communs fusionnés et transformés) avec accès aux hautes études assez limité : écoles supérieures techniques spécialisées, faculté de physique et chimie, de médecine (sous réserve).

4. Voir la note précédente.

5. On trouve souvent cette dernière «étiquette " chez les historiens à l'époque soviétique. Voir, par exemple, Ganelin 1950. La désapprobation de la réforme "tolstovienne » chez les historiens du début $\mathrm{du} \mathrm{XX}^{\mathrm{e}}$ siècle est plus nuancée, elle se limite aux transformations des gymnases, sans contester son essence et l'utilité générale. Voir Alešincev 1912 : 281-318.

6. Sergei Uvarov, le nouveau ministre de l'Instruction nationale (1833-1849), formulait l'idée directrice du fonctionnement du système éducatif en trois mots "pravoslavie, samoderžavie, narodnost » [foi orthodoxe, autocratie, authenticité nationale] qui caractérisent en général le règne (1825-1855) de Nicolas $\mathrm{I}^{\mathrm{er}}$.

7. La langue russe en tant que matière d'enseignement figure dans les programmes de gymnases à partir de 1811. La leçon a une durée variable : 1h30 jusqu'à 1828, 1h15 en 1864 et une heure à partir de 1872.

8. L'histoire de ce Comité Savant (ou Scientifique) se divise en deux périodes d'existence (1817-1831 ; 1856-1917) ; entre 1831 et 1856 , ses activités étant suspendues, la lecture critique des ouvrages pour l'école faisait partie des charges d'enseignants universitaires. Voir Georgievskij 1902.

9. Les périodiques de la seconde moitié du XIX ${ }^{\mathrm{e}}$ siècle les plus lus : Vestnik vospitanija (1890-1917), Gimnazia (1888-1900), Pedagogičeskij sbornik (1864-1918), etc.

10. 43 avis des savants et pédagogues étrangers furent réunis et publiés par le Ministère dans l'un des six volumes des remarques et avis sur les projets de réformes: Zamečanija inostrannyx pedagogov na proekty ustavov [Remarques des pédagogues étrangers sur les projets des statuts et des programmes d'études], Saint-Pétersbourg, 1863.

11. Voir par exemple le recueil annuel des travaux de cette section Pedagogičeskij Muzej voennoučebnyx zavedenij. Trudy cobranij prepodavatelea jazykov russkogo i inostrannyx. Année scolaire 1886-1887 [Musée pédagogique des établissements éducatifs militaires. Travaux d'enseignants des langues : russe et étrangères. Année scolaire 1886-1887], Saint-Pétersbourg, 1888.

12. C'est le cas de la revue officielle du Ministère de l'éducation «Žurnal Ministerstva NarodnogoPprosveščenia (ŽMNP), dont tous les numéros entre 1834 et 1908 sont actuellement mis en ligne.

13. Les méthodes d'apprentissage des langues modernes dans les établissements éducatifs fermés en font l'exception. Voir à ce sujet Kouzmina 2005.

14. Voir par exemple Perelogov Timothée. Etimologija, ili podrobnye nastavlenia o izmenenii slov francuzskoj reči. Izdanie dla upotreblenia $v$ etimologičeskix klassax $v$ gimnaziax pri imperatorskom Moskovskom universitete. [L'Étymologie, ou commentaires sur les modifications des parties du discours de la langue française. À l'usage des classes étymologiques du gymnase de l'université de Moscou]. Moscou, 1797 ; Vrais principes de la langue française, Moscou, 1804 ; Cours complet de langue française à l'usage de la jeunesse russe par Théodore Courtener. Seconde partie. Conjugaison, traduction et orthographe, Moscou, 1853. 
15. Cette expression « langue maternelle à travers les lunettes étrangères » lancée par Vladimir Dal (1801-1872) lexicographe éminent et slavophile, a été reprise et reproduite dans les articles polémiques de l'époque pour désigner tous les ouvrages de grammaire systématiques.

16. Greč N. Praktičeskaja grammatika [La grammaire pratique] Saint-Pétersbourg, 1827; 1834 ; Načalnye pravila russkoj grammatiki [Principes élémentaires de la grammaire russe] Saint-

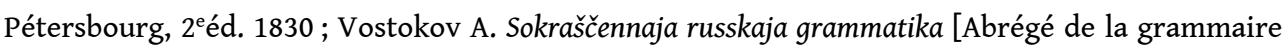
russe] Saint-Pétersbourg, 1828 ; Russkaja grammatika [Grammaire russe], Saint-Pétersbourg, 1831.

17. Buslaev F. I. O prepodavanii otečestvennogo jazyka, Moscou, 1844. Nous avons consulté cet ouvrage dans le livre réunissant plusieurs des travaux du savant y compris ses programmes d'études pour les différents établissements secondaires. Ayant le même titre, ce recueil a été publié en 1992 à Moscou, par les enseignants chercheurs de l'Université Lomonossov, Protčenko Ivan et Xodakova Ludmila: Buslaev F.I. O prepodavanii otečestvennogo jazyka. Učebnoe posobiedla pedagogičeskix universitetov. [Buslaev F.I. À propos de l'enseignement de la langue maternelle. Recueil des travaux en didactique. Pour les universités pédagogiques] Moscou: Prosveščenije, 1992. Les références aux autres écrits de Buslaev dans cet article renvoient aux pages de ce même recueil.

18. Les citations tirées de l'ouvrage de Buslaev sont traduites du russe par nos soins.

19. À son époque, ce n'est pas improbable car le recours aux services de précepteurs étrangers dans l'éducation des enfants est toujours de règle dans les milieux nobles et aisés. Au sein des gymnases le relai est souvent assuré par les natifs de français et d'allemand recrutés à ses fins (surveillants, maîtres/maîtresses de classes); c'est valable aussi pour les pensionnaires des établissements fermés de filles nobles; des corps des cadets. Pourtant ce ne sont pas les préalables garantis, surtout avec une démocratisation du public d'apprenants croissante dans la seconde moitié du XIX ${ }^{\mathrm{e}}$ siècle.

20. Cette idée de Buslaev trouve son incarnation officialisée dans les années 1870: l'enseignement du russe et du latin au niveau élémentaire sera principalement confié à la même personne.

\section{RÉSUMÉS}

L'apport de Fedor Buslaev (1818-1897), linguiste et pédagogue dans l'enseignement de la langue maternelle et des langues étrangères à l'école secondaire. L'essence de la nouvelle approche, mettant au centre le développement de l'activité langagière des élèves, fondement nécessaire pour la maîtrise consciente de tout idiome ainsi que la réception par la communauté pédagogique et savante russe au XIXe siècle.

Contribution of Fedor Buslaev (1818-1897), linguist and pedagogue in the teaching of the mother tongue and foreign languages in secondary school. The essence of new approach, focusing on the development of students' language activity, a necessary foundation for the conscious mastery of any idiom as well as reception by the Russian educational and scholarly community in the 19th century. 
INDEX

Keywords : Fedor Buslaev, linguist, Russia, 19th century, grammar, conscious, contrastive approach

Mots-clés : Fedor Buslaev, linguiste, Russie, XIXe siècle, grammaire, approche consciente, contrastive

\section{AUTEUR}

NADÉJDA KRIAJEVA

Université Clermont-Auvergne 